\title{
Preparation and Characterization of Poly(2-oxazoline) Micelles for the Solubilization and Delivery of Water Insoluble Drugs
}

Natasha Vinod ${ }^{1}, 2$, Duhyeong Hwang1, Salma H. Azam³, Amanda E. D. Van Swearingen³, Elizabeth Wayne $^{1}$, Sloane Christian Fussell ${ }^{4}$, Marina Sokolsky-Papkov ${ }^{1}$, Chad V. Pecot ${ }^{3,5,6}$ and Alexander V. Kabanov ${ }^{1,7, *}$

${ }^{1}$ Center for Nanotechnology in Drug Delivery and Division of Pharmacoengineering and Molecular Pharmaceutics, Eshelman School of Pharmacy, University of North Carolina at Chapel Hill, NC, USA; ${ }^{2}$ Joint UNC/NC State Department of Biomedical Engineering, University of North Carolina, Chapel Hill, NC, USA; ${ }^{3}$ Lineberger Comprehensive Cancer Center, University of North Carolina at Chapel Hill, Chapel Hill, NC, USA; ${ }^{4}$ Department of Biology, Department of Chemistry, University of North Carolina at Chapel Hill, Chapel Hill, NC, USA; ${ }^{5}$ Division of Hematology \& Oncology, University of North Carolina at Chapel Hill, Chapel Hill, NC, USA; ${ }^{6}$ Department of Medicine, University of North Carolina at Chapel Hill, Chapel Hill, NC, USA; ${ }^{2}$ Laboratory of Chemical Design of Bionanomaterials, Faculty of Chemistry, M.V. Lomonosov Moscow State University, Moscow, Russia

*For correspondence: kabanov@email.unc.edu

[Abstract] Many new drug development candidates are highly lipophilic compounds with low water solubility. This constitutes a formidable challenge for the use of such compounds for cancer therapy, where high doses and intravenous injections are needed (Di et al., 2012). Here, we present a poly(2oxazoline) polymer (POx)-based nanoformulation strategy to solubilize and deliver hydrophobic drugs. POx micelles are prepared by a simple thin-film hydration method. In this method, the drug and polymer are dissolved in a common solvent and allowed to mix, following which the solvent is evaporated using mild heating conditions to form a thin film. The micelles form spontaneously upon hydration with saline. POx nanoformulation of hydrophobic drugs is unique in that it has a high drug loading capacity, which is superior to micelles of conventional surfactants. Moreover, multiple active pharmaceutical ingredients (APIs) can be included within the same POx micelle, thereby enabling the codelivery of binary as well as ternary drug combinations (Han et al., 2012; He et al., 2016).

Keywords: Lipophilic, Poly(2-oxazoline), Nanoformulation, Surfactants, Active pharmaceutical ingredients, Polymeric micelles

[Background] Recent statistics show that only $3.7 \%$ of the new drug candidates that enter clinical testing are approved for use in cancer treatment. This has been primarily attributed to the poor pharmacokinetics of poorly water-soluble drug candidates, which results in suboptimal performance (Gala et al., 2020). POx polymeric micelles offer several advantages over traditional drug delivery systems such as liposomes, microparticles, and nanogels, among others. The unparalleled high solubilizing capacity of POx micelles for a large variety of hydrophobic drugs enables the delivery of greater amounts of drugs with a substantially lesser amount of excipient (Luxenhofer et al., 2010, He 
et al., 2016). POx-based drug formulations are easy to prepare, safe, and stable. Additionally, a quantitative structure-property relationship (QSPR) model has been developed to predict drug loading into POx micelles, which can be utilized to facilitate high throughput screening of sparingly soluble drug development candidates for incorporation in POx micelles (Alves et al., 2019).

\section{Materials and Reagents}

1. PVC tubing (Nalgene, $1 / 4 "$ ID)

2. Eppendorf tubes (Fisher Scientific, catalog number: 05-408-129)

3. Pipette tips (Fisher Scientific)

4. $11 \mathrm{~mm}$ plastic autosampler vials (Thermo Scientific, catalog number: C4011-13)

5. UV cuvettes (Fisher Scientific, catalog number: NC0628994)

6. 0.2-micron syringe filter, Nylon (Fisherbrand, catalog number: 13100108)

7. Poly(2-oxazoline) triblock copolymer (P[MeOx $\left.37-b-B u O x_{23}-b-M e O x_{37}\right]-$ piperazine) was synthesized as described previously (Luxenhofer et al., 2010)

8. Drugs were purchased from either Adooq Bioscience, Apex Bio, or LC laboratories and stored at $-20{ }^{\circ} \mathrm{C}$

9. Ethanol 200 proof (Fisher Scientific)

10. Normal Saline (Teknova, catalog number: S5815)

\section{Equipment}

1. Pipettes (Fisher Scientific)

2. Sonicator (Branson 2510 ultrasonic bath)

3. Eppendorf heating block (Fisher Scientific, catalog number: 11-715-1250)

4. Desiccator (LabCorp)

5. Rotor vacuum evaporator (Buchi)

6. Vortex mixer (Fisher Scientific, catalog number: 02215365)

7. Benchtop microcentrifuge (Thermo Scientific)

8. Eppendorf Centrifuge Minispin (Fisher Scientific, catalog number: 05-090-100)

9. HPLC (Agilent 1200 series)

10. Zetasizer (Malvern)

\section{Procedure}

A. Preparation of drug-loaded POx micelles (Figure 1, Small, $\mu \mathrm{L}$ scale; applicable to single and multidrug loaded POx micelles)

1. Prepare stock solutions of the drug and polymer in a common solvent. 
Note: Commonly used solvents for the preparation of POx micelles are ethanol (200 proof), methanol, and acetone. In some cases, a mixture of two solvents is used to dissolve drugs. The concentration of the stock solution is determined by the solubility of the drug/polymer in the solvent. E.g., Prepare Paclitaxel stock solution in ethanol at a concentration of $10 \mathrm{mg} / \mathrm{ml}$ and POx stock solution in the same solvent at a concentration of $10 \mathrm{mg} / \mathrm{ml}$ (POx is also soluble in acetone and methanol at $\geq 10 \mathrm{mg} / \mathrm{ml}$ ). You will need to use an ultrasonic bath for the dissolution of PTX in ethanol.

2. Mix the polymer and drug solutions at a predetermined polymer/drug ratio (w/w). Note: Polymer:drug weight ratio of 10:2 is a good starting point to determine if the polymer can solubilize the drug. If the thin film disperses well in saline (step 4), work your way up to higher ratios (10:4 and 10:8). E.g., for a 10:4 loading ratio of POx:PTX, add a $50 \mu$ stock solution of POx with a $20 \mu \mathrm{L}$ stock solution of PTX and mix using a vortex mixer (setting 10; for $\sim 10 \mathrm{~s}$ ).

3. Evaporate the solvent by placing the Eppendorf tube containing the drug-polymer mixture in a heating block and blowing a stream of nitrogen gas into the tube.

The appearance of a clear (not cloudy) thin film is indicative of optimal heating conditions (see Figure 2A). The optimum temperature for evaporation is dictated by the physicochemical properties of the solvent (e.g., vaporization temperature) and drug (e.g., Lipophilicity, polarity, etc.) in use. Of note, the optimized temperature of evaporation for Resiquimod and PTX is $50^{\circ} \mathrm{C}$ and $45{ }^{\circ} \mathrm{C}$, respectively, and for other drugs varies from $40{ }^{\circ} \mathrm{C}$ to $80^{\circ} \mathrm{C}$.

Note: A disposable pipette tip can be inserted into the end of a PVC tubing and lowered into the Eppendorf tube to facilitate a gentle flow of nitrogen. Further, residual traces of solvent can be removed by placing the thin film in the desiccator overnight.

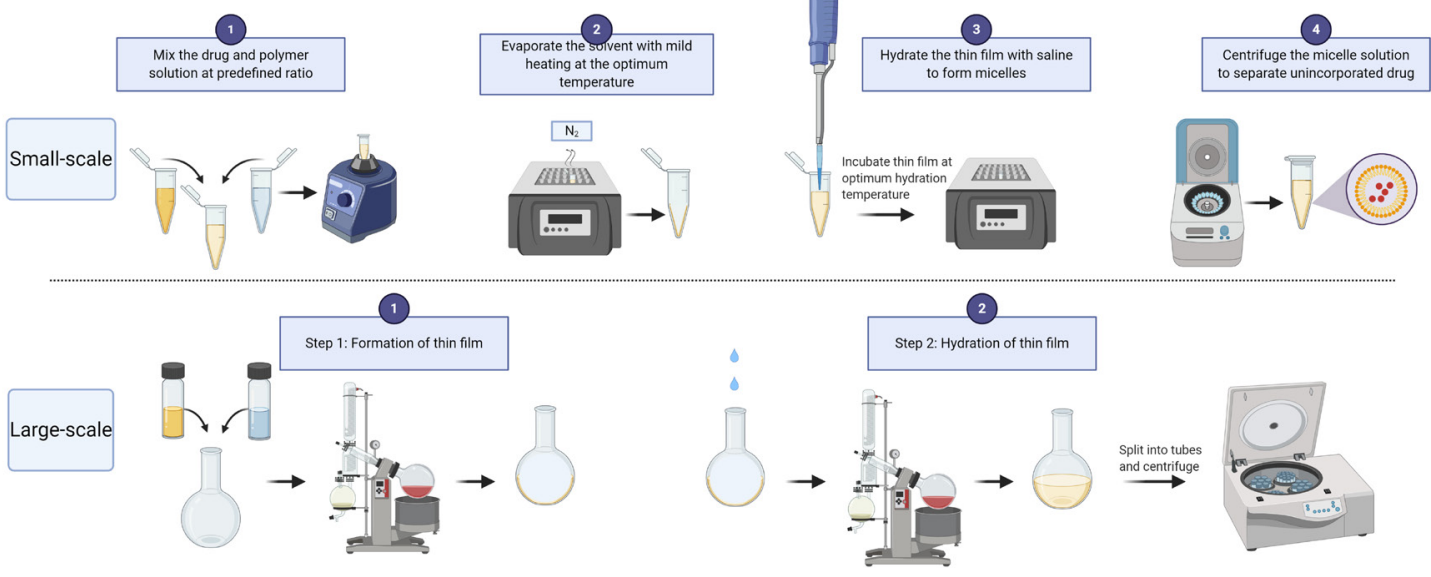

Figure 1. POx micelle preparation workflow (created with BioRender.com)

4. Hydrate the thin film with normal saline.

The volume of saline to be used is determined by the desired final drug(s) concentration. The optimal conditions of hydration vary with each drug. For instance, incubation for $10 \mathrm{~min}$ at RT 
followed by mild agitation by gently flicking the tube is sufficient for solubilizing thin films of Resiquimod. In comparison, Paclitaxel requires incubation at $65{ }^{\circ} \mathrm{C}$ for 20 min and gentle agitation every $5 \mathrm{~min}$. The temperature of hydration should be optimized empirically for every single and multi-drug incorporated POx micelles.

Note: Gentle agitation (using a vortex mixer or gently flicking the tube) is necessary to completely disperse the thin polymer-drug film. Visual inspection is a primary means of telling if the hydration conditions worked. While most drugs solubilize within $10 \mathrm{~min}$, for certain drugs, an additional incubation time can yield better size distribution (Figure 2C) by facilitating specific interactions between the drug and polymer (hydrophobic, hydrogen bonding, etc.)

5. Centrifuge the micelle solution at $10,000 \times g$ for 2-3 $\mathrm{min}$ to separate the unencapsulated drug (pellet) and transfer the supernatant containing the drug-loaded micelles to a new tube.

6. Depending on the stability of the POx micelle solution of drugs, these can either be stored at $4{ }^{\circ} \mathrm{C}$ for up to a couple of weeks or freeze-dried for long-term storage. The lyophilized formulation of POx micelles can be easily re-dispersed in DI water without loss in drug-loading and activity.

B. Preparation of drug-loaded POx micelles (Figure 1, large, $\mathrm{mL}$ scale; applicable to single and multidrug loaded POx micelles)

1. Follow Steps $A 1$ to $A 2$. Use glass vials to accommodate large volumes of stock solutions and a round-bottom (RB) flask for mixing the drug and polymer solutions.

2. Evaporate the solvent using a rotary vacuum evaporator. Adjust the temperature of the water bath as needed.

3. The thin film will form in the bottom half of the RB flask when completed.

4. Hydrate the thin film with saline and immerse it in the water bath at the optimal hydration temperature for the required time - usually 10 to $15 \mathrm{~min}$.

5. The micelle solution can be aliquoted into small volumes and centrifuged as in Step A5.

6. Lyophilize the formulation for long-term storage.

C. Characterization of POx micelles

The primarily used physicochemical characterization techniques for POx micelles include dynamic light scattering (DLS) for the determination of the size distribution and reverse-phase highperformance liquid chromatography for drug loading.

1. Sample Preparation for DLS measurement

a. Dilute the micelles 10 -fold in saline.

Note: Typical dilutions used for DLS measurements of POx micelles range from 1:2-1:50, which correspond to drug concentrations of $0.1 \mathrm{mg} / \mathrm{ml}$ to $2 \mathrm{mg} / \mathrm{ml}$.

b. Use nylon syringe filters of 0.2 microns to separate large particles.

c. Transfer the filtered solution into a clean cuvette without introducing air bubbles.

d. Place the cuvette in the sample holder and, following equilibration for at least 2 min, take 3 
separate measurements for every sample.

\section{Sample preparation for HPLC measurement}

a. Dilute the micelle sample 50 -fold in a mixture of 50/50 acetonitrile (ACN): water (v/v).

b. Filter the diluted sample using a 0.2 -micron syringe filter and transfer $100 \mu$ into an HPLC autosampler vial.

c. Get rid of any air bubbles from the autosampler vial by gently tapping before placing it in the vial tray.

d. Prepare the standards by serially diluting the analyte at concentrations ranging from 6.25 $\mu \mathrm{g} / \mathrm{ml}$ to $200 \mu \mathrm{g} / \mathrm{ml}$.

e. The run settings must be optimized for each drug

Note: 60/40 ACN: water, $1 \mathrm{mg} / \mathrm{ml}$ flow rate, and $10 \mu$ injection volume is usually a good starting point for most drugs. Spiking the mobile phase solvents with $0.1 \%$ trifluoroacetic acid helps sharpen the peaks. Make sure the settings used for the sample are identical to the ones used for standards.

\section{Data analysis}

\section{A. DLS Analysis}

The zeta sizer measures fluctuations in the scattered light intensity with time caused by the Brownian motion of nanoparticles to estimate the hydrodynamic size of nanoparticles. DLS analyzes the raw data using two algorithms, viz., cumulant analysis and distribution analysis. The cumulant analysis reports two values: Z-average, which is the mean value of the particle size distribution, and polydispersity index (PDI), which is analogous to variance. A PDI value lower than 0.2 is indicative of monodisperse particles. The cumulant analysis also provides a correlogram (correlation curve), which reports the decay rate of the signal from the sample. The amplitude of the correlogram is representative of signal to noise ratio. The distribution analysis provides Gaussian distributions of the particle size by number, volume, and intensity. Sample concentration, presence of large aggregates, and impurities or marks on the exterior of the DLS cuvettes can all influence the size distribution. More information about data interpretation can be found at https://www.malvernpanalytical.com. 


\section{bĭo-protocol}
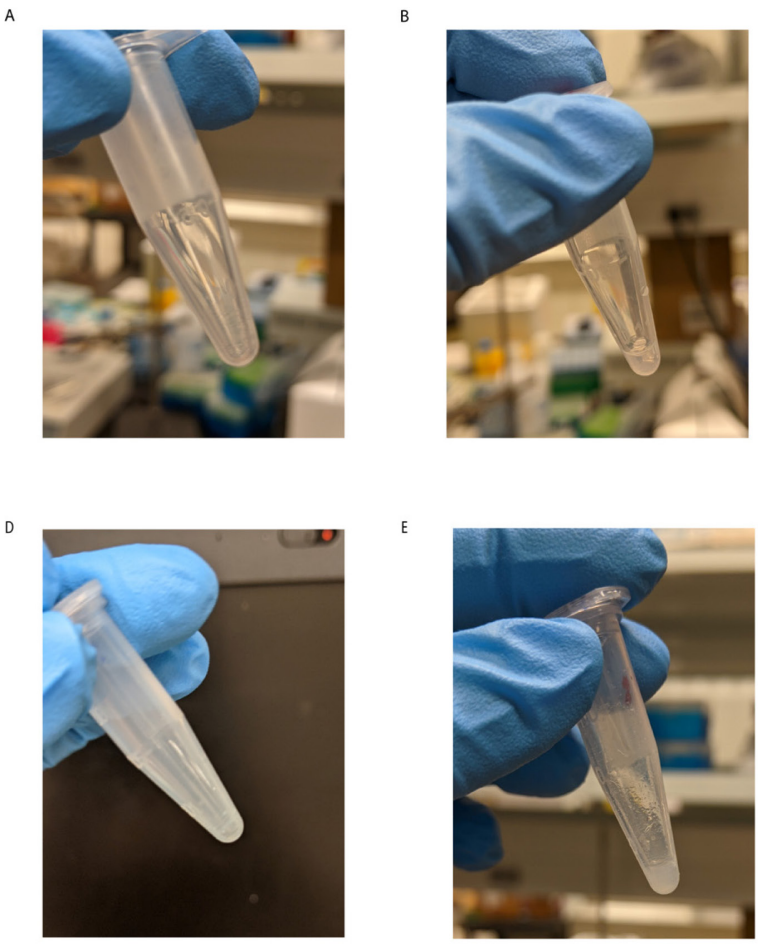
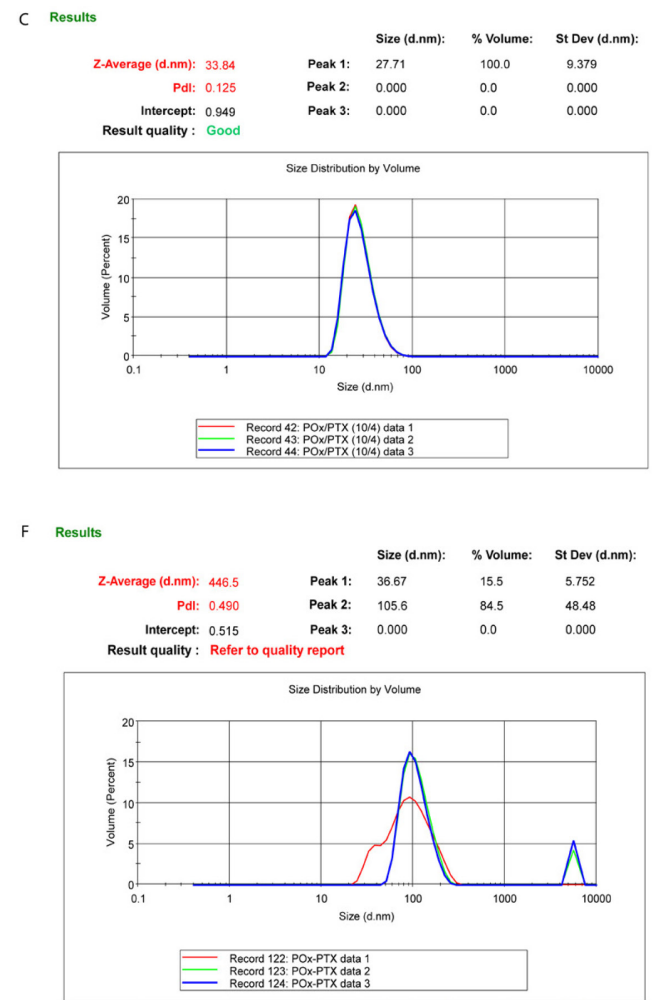

Figure 2. Representative images of the (A, D) thin film, (B, E) hydrated thin film, and (C,

F) DLS volume distribution. The top panel represents drug-incorporated micelles prepared using optimal conditions, as demonstrated by the monodisperse volume distribution with a single peak around $27 \mathrm{~nm}$. The bottom panel is representative of sub-optimal conditions, indicated by the cloudy thin film and the incomplete hydration of the thin film, resulting in polydisperse size distribution with a peak at about $105 \mathrm{~nm}$ and a large peak of aggregates in the micrometer range.

Note: The DLS data were obtained from a separate experiment and do not represent the size distribution of the micelles shown in the images and is used here for demonstration purpose only.

\section{B. Drug Loading}

Chose the wavelength at which the analyte shows maximum absorbance and then integrate the peak in the chromatogram to obtain the area, which corresponds to the concentration of the drug in the sample. The retention time of the sample should compare to that of standards. Once the sample concentration is determined from the standard curve, parameters such as loading efficiency and loading capacity can be obtained from the following equations:

$$
\begin{aligned}
& \mathrm{LC}=\left(\frac{M_{\mathrm{drug}}}{M_{\text {drug }}+M_{\text {excipient }}}\right) \times 100 \\
& \mathrm{LE}=\left(\frac{M_{\text {drug }}}{M_{\text {drug added }}}\right) \times 100
\end{aligned}
$$


Mdrug: The amount of drug incorporated in the micelle

Mexcipient: The amount of polymer used in the formulation

Mdrug added: The amount of drug initially fed

\section{Acknowledgments}

The original work (Vinod et al., 2020) was funded by the National Cancer Institute (NCl) Alliance for Nanotechnology in Cancer (U54CA198999, Carolina Center of Cancer Nanotechnology Excellence).

\section{Competing interests}

A. V.K. is co-inventor on patents pertinent to the subject matter of the present contribution and A.V.K. and M.S.P. have co-founders' interest in DelAqua Pharmaceuticals Inc. having intent of commercial development of POx based drug formulations. The other authors have no competing interests to report.

\section{References}

1. Alves, V. M., Hwang, D., Muratov, E., Sokolsky-Papkov, M., Varlamova, E., Vinod, N., Lim, C., Andrade, C. H., Tropsha, A. and Kabanov, A. (2019). Cheminformatics-driven discovery of polymeric micelle formulations for poorly soluble drugs. Sci Adv 5(6): eaav9784.

2. Di, L., Fish, P. V. and Mano, T. (2012). Bridging solubility between drug discovery and development. Drug Discov Today 17(9-10): 486-495.

3. Gala, U. H., Miller, D. A. and Williams, R. O., 3rd (2020). Harnessing the therapeutic potential of anticancer drugs through amorphous solid dispersions. Biochim Biophys Acta Rev Cancer 1873(1): 188319.

4. Han, Y., He, Z., Schulz, A., Bronich, T. K., Jordan, R., Luxenhofer, R. and Kabanov, A. V. (2012). Synergistic combinations of multiple chemotherapeutic agents in high capacity poly(2-oxazoline) micelles. Mol Pharm 9(8): 2302-2313.

5. He, Z., Wan, X., Schulz, A., Bludau, H., Dobrovolskaia, M. A., Stern, S. T., Montgomery, S. A., Yuan, H., Li, Z., Alakhova, D., Sokolsky, M., Darr, D. B., Perou, C. M., Jordan, R., Luxenhofer, R. and Kabanov, A. V. (2016). A high capacity polymeric micelle of Paclitaxel: Implication of high dose drug therapy to safety and in vivo anti-cancer activity. Biomaterials 101: 296-309.

6. Luxenhofer, R., Schulz, A., Roques, C., Li, S., Bronich, T. K., Batrakova, E. V., Jordan, R. and Kabanov, A. V. (2010). Doubly amphiphilic poly(2-oxazoline)s as high-capacity delivery systems for hydrophobic drugs. Biomaterials 31(18): 4972-4979.

7. Vinod, N., Hwang, D., Azam, S. H., Van Swearingen, A. E. D., Wayne, E., Fussell, S. C., Sokolsky-Papkov, M., Pecot, C. V. and Kabanov, A. V. (2020). High-capacity poly(2-oxazoline) formulation of TLR $7 / 8$ agonist extends survival in a chemo-insensitive, metastatic model of lung 
adenocarcinoma. Sci Adv 6(25): eaba5542. 